\title{
Protest in der Gesellschaft und Kritik in der Soziologie
}

\section{Gerda Bohmann}

Zusammenfassung: Gegenstand des Beitrags ist die neuerlich zur Diskussion stehende Beziehung von Soziologie und Gesellschaftskritik. Diese wird aber nicht im Rahmen einer kritischen theoretischen Auseinandersetzung behandelt, sondern vor dem Hintergrund konkret-praktischer Äußerungsformen von Gesellschaftskritik durch unterschiedliche gesellschaftliche Gruppen mit gegensätzlichen Anliegen. Konkret geht es um die Frage des Verhältnisses (kritischer) Soziologie zu den neuesten Protestbewegungen.

Schlüsselwörter: Soziologie, Gesellschaftskritik, neue soziale Bewegungen

\section{Protest in society and criticism in sociology}

\begin{abstract}
The paper focusses on the renewed discussion about the relation between sociology and the critique of society. But in the center of my interest is not the critical debate of this discussion, but rather it's background, the concrete and practical forms of critique in society by different social groups formulating antagonistic aims and purposes. Concretely, I am concerned with the question about the relation between (critical) sociology and the newest social movements.
\end{abstract}

Keywords: sociology, critique of society, new social movements

G. Bohmann $\bowtie$

WU - Wirtschaftsuniversität Wien, Institut für Soziologie und Empirische Sozialforschung Welthandelsplatz 1, Gebäude D4, 1020 Wien, Österreich

E-Mail: Gerda.Bohmann@wu.ac.at 
Nachdem es um die Gesellschaftskritik in der Soziologie längere Zeit ruhig geworden war, sind im neuen Jahrtausend, freilich insbesondere im Zusammenhang mit den tiefgreifenden Krisen des globalisierten Kapitalismus und den sich verschärfenden sozialen Ungleichheiten, gleich mehrere prominente Beiträge und Stellungnahmen zur gesellschaftskritischen Funktion der Soziologie publiziert worden. (vgl. Dörre, Lessenich und Rosa 2009; Boltanski 2010; Vobruba 2009; Jaeggi und Wesche 2009; u.a.m.) Diese Beiträge zielen nicht mehr auf die Totalität der gesellschaftlichen Verhältnisse, wie noch in den siebziger Jahren des zwanzigsten Jahrhunderts, werfen aber erneut die Frage nach der inneren Beziehung zwischen Soziologie und gesellschaftlicher Aufklärung, bzw. zwischen Soziologie und Kritik auf. Es geht mir in diesem Beitrag nicht so sehr darum, mich diesen neuen Ansätzen kritisch zu nähern, sondern vielmehr um die Frage nach gesellschaftlichen Akteuren, die Kritik an den gegenwärtigen Verhältnissen üben, und zwar in der Form von Protestbewegungen, die ich als Ausdruck praktischer Gesellschaftskritik betrachte. Anders gesagt: ich werde die Frage, was Kritik in der Soziologie angesichts von Protest in der Gesellschaft bedeuten kann, vor dem Hintergrund zweier widersprüchlicher Beispiele sozialer Protestbewegungen bearbeiten, dem so genannten Arabischen Frühling, d.h. jener Protestbewegung, die im Jahr 2011 zum Sturz zweier autokratischer Staatschefs geführt hat, weit in die arabische Welt ausgestrahlt ist und auch die „Occupy-Bewegung“, insbesondere „15 M“ in Spanien inspiriert hat (1.); und die sich 2014 in Deutschland formierende rechtspopulistische Bewegung mit Pegida, Legida u.a.m. (2.), die ebenfalls Nachahmer in anderen europäischen Ländern gefunden hat.

\section{Protest in der Gesellschaft}

1. Beispiel: Jasmin-Revolte, Arabischer Frühling, oder Protest im Namen von „Brot (bzw. Leben), Freiheit, menschlicher Würde“1

Wenngleich es in der arabischen Welt auch in den Jahrzehnten davor Gründe für Protest und Revolte gegeben hatte und sich durchaus in gewerkschaftlichen Aktivitäten sowie jenen der politischen Opposition niedergeschlagen hat, so kam der Aufstand der revoltierenden Jugend Ende 2010 und Anfang 2011 doch überraschend. Der Anlass war, wenngleich tragisch, so doch weitgehend zufällig: die Selbstverbrennung des arbeitslosen Gemüsehändlers Muhammad Bu

1 „Einer der Slogans, der bei den Demonstrationen in Ägypten skandiert wurde, lautete, , aish, hurriya, karama insaniyya! - auf Deutsch: Brot, Freiheit, menschliche Würde! Im ägyptisch-arabischen Dialekt ist'aish das Brot, in der arabischen Schrift- und Hochsprache ist es das Leben; tatsächlich ist Brot in Ägypten das Grundlebensmittel gerade für die Armen." (Perthes 2011, S. 25 Hervorhebungen im Original) 
Azizi am 17.12.2010 (und kurz danach eine weitere Selbsttötung²) in Tunesien. Unmittelbar nach dessen Tod versammelten sich vor dem Amtssitz des Gouverneurs zunächst einige Jugendliche; nach gewaltsamen Zusammenstößen mit der Polizei aber immer mehr und mehr Menschen, die sich durch gewaltsames Eingreifen der Polizei nicht mehr vertreiben ließen. Die Demonstrationen dauerten über den Jahreswechsel hinweg an, bis schließlich am 14. Jänner 2011 der seit $1987 \mathrm{im}$ Amt befindliche autokratische Präsident Zine el-Abidine Ben Ali mitsamt seiner Familie aus dem Land flieht. ${ }^{3}$

Ägypten war das erste Land, auf das der tunesische Aufstand übergegriffen hat. Entscheidend sollte hier der 25. Januar 2011, der „Tag der Polizei“, werden. Zunächst ging es den Demonstrantinnen um Menschenwürde und Rechte, die Regimefrage wurde vorerst noch gar nicht gestellt; aber auch das sollte angesichts der (in einer zweiten Phase) repressiven und gewaltsamen Reaktion des Regimes dann sehr schnell gehen.

„Organisatoren und andere Zeitzeugen sind sich einig, dass die Beteiligung an der Demonstration alle Erwartungen überstieg. Einige, [...], hätten von 20.000 Teilnehmern geträumt, aber niemand habe die 60.000 bis 70.000 für möglich gehalten, die sich an diesem Tag wohl tatsächlich versammelten.“ (Perthes 2011, S. 54)

Eine Woche nach Beginn der Proteste (am 1. Februar) wurde zum „Marsch der Millionen“ aufgerufen, dem diese v.a. in den großen Städten auch folgten. Ohne hier auf die Chronologie der wechselvollen Ereignisse eingehen zu können (vgl. dazu: Nordhausen 2011), soll eine längere Passage aus dem Text von Volker Perthes über die „18 Tage, die die arabische Welt erschütterten" wiedergegeben werden:

„[...] am ersten Tag wurde offenkundig, dass das Regime das Ausmaß des Protests unterschätzt hatte. Zwar gelang es den Sicherheitskräften noch, den Tahrir-Platz, der zum zentralen Ort der Revolte werden sollte, zu räumen. Das tat der Kraft dieser ersten großen Demonstration keinen Abbruch. Der folgende Freitag, der 28. Januar, wurde von der Protestbewegung zum ,Tag des Zorns' erklärt. Er wurde dies auch, nicht nur in Kairo, sondern in fast allen größeren Städten des Landes. Die Regierung versuchte, die Proteste einzudämmen, indem sie Internet und Mobilfunk lahmlegte. Vergeblich, denn der Protest ging mittlerweile weit über jene hinaus, die Facebook-Aufrufe lasen. Zahlreiche Menschen gingen direkt nach den Freitagsgebeten auf die Straße. Aus den Demonstrationen der Mittelschichtangehörigen war eine Massenbewegung geworden.“ (Perthes 2011, S. 55)

2 „Am 22. Dezember griff in Sidi Bouzid der 24-jährige arbeitslose Houcine Néji demonstrativ in die Hochspannungsleitung, nachdem er noch ,Arbeit für alle! Weg mit Ben Ali!' Geschrien hatte.“ (Schmid 2011, S. 16)

3 Zur Chronologie der Ereignisse vgl. Schmid 2011; Perthes 2011 u.a.m. 
Waren Vertreter der Muslimbrüder zu Beginn als einfache Demonstrationsteilnehmer, nicht als Organisation, unter den Hunderttausenden, die sich in Kairo versammelten, so unterstützte die Muslimbruderschaft nach den Straßenschlachten mit der Polizei, bei denen diese die Kontrolle verlor, die Proteste. (vgl. Perthes ebd. und f.; Nordhausen 2011, S. 48) Diese Unterstützung ist, Perthes zufolge, aufgrund deren landesweiten Organisation und ihrer Erfahrung in Auseinandersetzungen mit der Polizei essentiell gewesen. Wie schon Ben Ali zuvor, musste auch Staatschef Hosni Mubarak (im Amt seit 1981) am 11. Februar zurücktreten.

Wenngleich diese „Arabellion“ von 2011 mittlerweile als gescheitert gilt, die „[...] Hoffnung auf Würde, Freiheit, Wohlstand und vielleicht auch Demokratie [...] sich nicht erfüllt [hat]" (Gerlach 2016, S. 47), so schien mit dem politischen Umsturz in Tunesien und in Ägypten auch der soziale Umbruch eingeleitet zu sein.

Die Rolle der sozialen Netzwerke für die Verbreitung und Mobilisierung in der Anfangsphase des Aufstands ist unbestritten, aber weder dies noch der unmittelbare Anlass des Aufstands kann als dessen Ursache betrachtet werden. Neben der Rolle der neuen Medien (einschließlich des Satellitenfernsehen, v.a. al-Jazeera), weltwirtschaftlichen, politischen und sozialstrukturellen Faktoren dürfte ,[...] die wichtigste erklärende Variable für den Umbruch [...] aber in der demographischen Entwicklung liegen.“ (Perthes 2011, S. 24)

Perthes nennt die zwischen 1975 und 1990 Geborenen, die i.d.R. über 30\% der Gesamtbevölkerung stellen, die Baby-Boomer der arabischen Welt (im Vgl. zu den 15 Jahrgängen davor - ebd., S. 30) ${ }^{4}$. Sie sind besser gebildet als die Generationen vor ihnen, sie sind besser vernetzt und es sind sie, die Zugang zum Internet haben, social media, Foren und mobile Kommunikationsmittel nutzen. „Quantitativ [...] gilt, dass dort in den letzten zwei Jahrzehnten der Anteil der Jugendlichen, die Zugang zu weiterführenden Schulen und Hochschulen erhalten, enorm gewachsen ist." (ebd., S. 31) Sie sind also qualifiziert, haben Zertifikate, finden aber keine Beschäftigung, was sich in sehr hohen Raten der Jugendarbeitslosigkeit niederschlägt. Wenngleich eine verlässliche Datenquelle dazu fehlt, ist davon auszugehen, dass sie in den arabischen Staaten höher ist als in jeder anderen Weltregion.

Überall in der arabischen Welt, wo der Arabische Frühling Resonanz gefunden hat, fanden sich in etwa dieselben Slogans und Forderungen, die sich von jenen der „neuen sozialen Bewegungen" in Europa deutlich unterscheiden, aber auch nicht mehr viel mit den islamistischen Bewegungen der sechziger und siebziger Jahre des zwanzigsten Jahrhunderts gemein haben. Sie sind jenen gegenüber als unideologisch, postislamistisch sowie anti-nationalistisch zu charakterisieren:

4 Emmanuel Todd und Youssef Courbage betonen, dass der Beginn des demographischen Wandels mit einem dramatischen Rückgang bei den Geburten (von über 7 auf 2-3) in Tunesien und Ägypten bereits zwischen 1965 und 1970 eingesetzt habe; mit einer zweiten Phase in Ägypten von 1985 bis 1990. (vgl. Courbage/Todd 2008, S. 89) 
„Actually, if you look at the people who launched these revolts, it is clear that they represent a ,post-Islamist generation'. For this generation, the great revolutionary movements of the 1970s and 1980s are history - something that mattered to their parents but not to them. This new generation is not interested in ideology, and their slogans are pragmatic and very concrete (like ,erhal', the Arabic word for ,get out'). They do not invoke Islam like the older generation did in Algeria in the late 1980s. What they mainly express is rejection of corrupt dictatorships and the demand for democracy." (Roy 2011, S. 1)

Volker Perthes bezeichnet sie auch als „eine skeptische Generation“, die sich ihrer Rolle als politischer Akteur bewusst gewesen ist. Die am weitesten verbreitete Parole „Das Volk will den Sturz des Systems" wurde allerorten mit den Forderungen nach Würde, Gerechtigkeit und Freiheit verknüpft. (Perthes 2011, S. 34 und vgl. ebd.) Deren Anliegen, deren Forderungen und deren Manifestationen können mit Fug und Recht als praktische Gesellschaftskritik verstanden werden.

2. Beispiel:„Pegida, Legida, etc.“ oder Protest im Namen von „Heimat, Frieden und deutscher Leitkultur" bzw. „Wir sind das Volk“"

„Plötzlich waren sie da, die ,Patriotischen Europäer gegen die Islamisierung des Abendlandes $^{`}$. Aus dem Nichts entstanden, brachten sie Tausende auf die Straße, um gegen ,Wirtschaftsflüchtlinge‘, Salafisten, Glaubenskriege und gegen die ,Lügenpresse` zu demonstrieren. [...] Waren auf der ersten Demonstration, zu der das ,Aktionsbündnis‘ am Montag, den 20. Oktober 2014, in Dresden aufrief, lediglich 350 Demonstranten erschienen, kamen vier Wochen später bereits 3.200 und weitere vier Wochen darauf, also am 15. Dezember, schon 15.000 ,patriotische Europäer', die bei nasskaltem Wetter in der Dunkelheit durch die Elbmetropole ,spazierten“.“(Geiges, Marg und Walter 2015, Position 1056) ${ }^{6}$

Mittlerweile hat auch diese Bewegung andere deutsche und europäische Städte erreicht. Aber ganz so eindeutig sind die Themen und Forderung dieser Gruppierung nicht; sämtliche seitdem publizierte Studien weisen darauf hin, dass die vordergründige Islamfeindlichkeit zwar „Zündfunke“ (Vorländer 2015), aber keineswegs das zentrale Thema der Protest-Spaziergänge gewesen sind. Vielmehr geht es um Kritik an der Einwanderungs- und Asylpolitik, Kritik an Politiker/innen und der Regierungspolitik generell, Unzufriedenheit mit der Berichterstattung der Medien und erst nachrangig um Islam, Kriminalität von Asylwerbern, Überfremdung usw.

5 http://www.europeaninstitute.org/index.php/ei-blog/119-february-2011/1238-qpost-islamic-revolutionqevents-in-egypt-analyzed-by-french-expert-on-political-islam - zuletzt abgerufen am 17.04.2016.

6 Die Positionsnummer bezieht sich auf die elektronische Buchausgabe (Kindle e-book). 
(vgl. WZB-Bericht 2015, 47 ff.); bzw. einen diffusen Hintergrund (...) Ängste in Bezug auf die Zukunft, Abstiegsängste, Angst vor Verlust des Arbeitsplatzes u.a.m. (vgl. Rucht 2014). Die Slogans sind allerdings (auch hier) recht einheitlich: „Lügenpresse, halt die Fresse“; „Das System ist am Ende, wir sind die Wende“; „Multikultur tötet“; Legida: „Für Heimat, Frieden \& deutsche Leitkultur. Gegen religiösen Fanatismus. Gegen Islamismus und Multikulti“ etc. (vgl. Geiges, Marg und Walter 2015)

Im Gegensatz zur Protestbewegung der arabischen Jugend ist die deutsche Bewegung von (überwiegend) rechts eine Protestbewegung des etablierten Mittelstandes: der typische PegidaDemonstrant ,[...] stammt demnach aus der Mittelschicht, ist gut ausgebildet und berufstätig, verdient etwas mehr als der durchschnittliche Sachse. Er ist ein Mann, gehört keiner Religion und keiner Partei an - und ist Sachse [...]" (Vorländer 2015; vgl. auch Pegida-Studie der TUDresden 2015; zit. in Spiegel-Online 2015 v. 14.01.2015). Zu ganz ähnlichen Schlüssen kommen die Berliner Studie sowie auch jene des Göttinger Instituts für Demokratieforschung:

„Die Quote der formal Hochgebildeten ist bei Pegida erheblich geringer [als bei Stuttgart 21 - Anm.]. Aber [...] das Proletariat repräsentieren die neuen Demonstranten von rechts nicht. [...] 35 Prozent der Befragten verfügen über einen Universitäts- und Fachhochschulabschluss, als Absolventen von Haupt- oder Volksschulen gaben sich unter den Teilnehmern der Umfrage lediglich 0,6 Prozent aus. Auch der Arbeiteranteil lag nur bei 7,1 Prozent, gegenüber Angestellten mit 37,3 Prozent und Freiberuflichen/Selbständigen mit 16,2 Prozent. 77 Prozent sind voll erwerbsfähig; 9,2 Prozent beziehen Rente. Einem sozial ausgegrenzten ,Prekariat" gehören die 500 [Anm.: hier befragten] Pedigisten nicht an.“ (Walter 2015)

Auch hinsichtlich der politischen Affinitäten handelt es sich nicht um eine homogene Gruppe, wenngleich an die $45 \%$ der AfD nahe stehen. (vgl. ebd.)

„[...] die hohe Emotionalität, der konfrontative Gestus, der Modus zur Schau gestellter Entrüstung und der erfolgreiche Versuch, kommunikative Macht auf prominenten Plätzen und Straßen zu erzeugen, [haben] Pegida zu einer Protestbewegung neuen Stils, einer rechtspopulistischen Empörungsbewegung, werden lassen. [...] Pegida wurde aber erst in dem Moment zu einer Bewegung, als sie prominente Straßen und Plätze, also den realen Raum, öffentlichkeitswirksam zu besetzen wusste. Weil sie es tat, vergrößerte sich die Teilnehmerschaft. Performativer Akt und Konstituierung als Massenbewegung gingen Hand in Hand.“ (Vorländer 2015a)

Ob diese nun als Sammlungsbewegung bezeichnet wird, die Widersprüchliches unter einem Dach vereint, „,...] in der Mitte im Trüben fischt und die Enttäuschten dieser Republik einsammelt $[\ldots]$ “. (Assheuer 2014), oder als neuer Typus sozialer Bewegungen im Kontext der 
Postdemokratie (Ullrich 2015) ${ }^{7}$ gelten kann - dass es sich um eine soziale Protestbewegung handelt, ist schwerlich in Zweifel zu ziehen; ebenso wenig deren gesellschaftskritisches Ansinnen, wenngleich dieses weniger revolutionär denn reaktionär, weniger progressiv denn regressiv erscheint.

\section{Soziale Protestbewegungen - soziologisch gerahmt}

Beim ersten Beispiel gibt es in der Soziologie vermutlich nicht nur breiten Konsens darüber, dass es sich um eine soziale Protestbewegung handelt, insbesondere weil sie sich Demokratie, Freiheit und Menschenwürde auf die Fahnen geschrieben hat, sondern tendenziell auch die Einschätzung, dass letztlich die Zivilgesellschaft ihre Stärke gezeigt habe. Sie ist nicht mit den „neuen sozialen Bewegungen“ der sechziger und siebziger Jahre des zwanzigsten Jahrhunderts gleichzusetzen, ihre Slogans sind deutlich zu unterscheiden. Aber sie entspricht doch weitgehend jenem „emanzipatorischen“ Typus von Bewegungen, dem die Protest- und Bewegungsforschung sich gewidmet hatte. Kontroversieller wird es wahrscheinlich beim zweiten Beispiel „postdemokratischer Empörung“(Ullrich 2015), bei der es zwar nicht schlicht um das Gegenteil geht, aber zumindest doch um Begrenzung des Zugangs zu den Errungenschaften der Demokratie, Freiheit und Würde für jene Menschen, deren kultureller und religiöser Hintergrund als fremd und bedrohlich angesehen wird. In der Begrifflichkeit von Wilhelm Heitmeyer haben wir es hier nicht zuletzt auch mit „gruppenspezifischer Menschenfeindlichkeit“ zu tun. Oder anders gesagt:

„Der Protest, der sich erstmal bei Pegida auf den Straßen und Plätzen deutscher Großstädte zeigte, musste [...] zwangsläufig als ,dunkle Seite' der Zivilgesellschaft gelten - insbesondere weil sich damit die Kritik an öffentlich artikulierten Formen von Intoleranz, Nationalpatriotismus und Fremdenfeindlichkeit verbindet.“(Vorländer 2015)

Aber beide Beispiele stehen für soziale Protestbewegungen. In beiden wird, durchaus fundamentale Kritik an der jeweiligen Gesellschaft geübt, an die sie sich adressiert; es wird deren Umbau im Sinne der je eigenen Ziele eingefordert. Sowohl aus einer Akteur-zentrierten Perspektive, in der soziale Bewegung sich als ,[...] kollektiver Akteur, mithin ein Netzwerk verschiedener anderer Akteure [fassen lässt], der auf Basis symbolischer Integration und eines gewissen Zugehörigkeitsgefühl (einer kollektiven Identität) mittels Protest sozialen Wandel erreichen, beschleunigen, verhindern oder umkehren will [...]" (Ullrich 2015, S. 9), als auch

7 Als ,postdemokratische Empörung“, wobei Peter Ullrich die „Einheit im Widerspruch“ von Occupy und Pegida behandelt. 
aus systemischer Perspektive, zumal diese Form der Mobilisierung von Empörung weder eine Organisation noch ein bloßes Interaktionssystem ist, ein soziales System aber freilich dennoch. Es lässt sich über seine Form fassen: den Protest; sein Medium ist die Protestkommunikation. Protestbewegungen, schreibt Luhmann, auch ,[...] die neueste neue soziale Bewegung [...]: die Bewegung der Ausländerfeinde, die nun auch jede Koalition mit den inzwischen klassischen Protestbewegungen aufkündigt [...]“(Luhmann 1996, S. 202) operieren „um der Sache willen“. Sie ,[...] argumentieren als ,Betroffene‘ für ,Betroffene ‘[...] die Protestkommunikation erfolgt zwar in der Gesellschaft, [...], aber so, als ob es von außen wäre. Sie äußert sich aus Verantwortung für die Gesellschaft, aber gegen sie.“ (a.a.O., S. 204) Dieser Typus eines sozialen Systems lässt sich auch als Konfliktsystem bezeichnen; und es lassen sich weitere Charakteristika benennen (vgl. Bergmann 1987, S. 362-372):

- Konfliktsysteme gehen in Opposition zur herrschenden Kommunikation, womit Erwartungssicherheit aufgelöst wird; der Widerspruch wird zum Auslöser sozialen Wandels.

- Sie gehen weder von einer Anfangsursache aus, noch von einem Zusammenschluss Einzelner, vielmehr von kontingenten Anlässen; und von da an entsteht Eigendynamik, „[...] die Bewegung bewegt die Bewegung sich selbst“". (ebd., S. 362)

- Es werden gesellschaftliche Strukturen thematisiert, was einen hohen Integrationseffekt nach innen, nach außen aber eine prinzipielle „Rücksichtslosigkeit“ bewirkt.

- Die relative Voraussetzungslosigkeit von Konfliktsystemen, die Kontingenz des Anfangs bedingt auch eine nicht-lineare Dynamik. Diese ist vielmehr abhängig von der jeweils erzeugten Resonanz. ${ }^{8}$

- Mit Niklas Luhmann lässt sich hinzufügen: „Der Protest inszeniert ,Pseudoereignisse“ [...], das heißt: Ereignisse, die von vorneherein für Berichterstattung inszeniert sind und gar nicht stattfinden würden, wenn es Massenmedien nicht gäbe [...]“ (Luhmann 1996, S. 212)

Mit je unterschiedlichen Schwerpunktsetzungen lassen sich die einzelnen Aspekte auf beide genannten Beispiele anwenden. In keinem der beiden Fälle lässt sich eine entscheidende Ursache erkennen, die zur Herausbildung der jeweiligen Protestbewegung geführt hat, vielmehr kontingente Anlässe, die auch andere hätten sein können, wenngleich sich freilich eine Vielfalt von Gründen für die Mobilisierung ausmachen lassen. In beiden Fällen ist es Empörung beziehungsweise Widerspruch gegenüber etablierten, insbesondere politischen Strukturen, die Menschen bewegt hat sich zu mobilisieren. Die sich entwickelnden Dynamiken sind in keinem der beiden Fälle voraussehbar oder steuerbar gewesen. Und schließlich ist die Rolle der neuen (und älteren)

8 Nicht die Ursache ist das Entscheidende, sondern der sich selbstselektiv entfaltende Konfliktprozess, die Ausbildung einer eigenen Selbstreferenz als Bewegung (vgl. Bergmann 1987, S. 371 f.) 
Medien sowohl in der Mobilisierung als auch in der jeweiligen Resonanz, die wiederum weitere Mobilisierung sowie deren Wirkungen bewirkt, kaum zu überschätzen.

\section{Protest, Gesellschaftskritik und die Soziologie}

Wie verhält sich aber nun die Soziologie zu diesen neuesten sozialen Bewegungen, bzw. wie stellt der Zusammenhang von Protest in der Gesellschaft und (Gesellschafts-)Kritik in der Soziologie sich dar? Sowohl aus einer akteurs- wie aus einer beobachtungszentrierten Perspektive kann Protest in Form sozialer Bewegungen als praktische Gesellschaftskritik gesehen werden. Im Rahmen der Theorie kommunikativen Handelns (Habermas 1981) entstehen Protestpotentiale da, wo Systemimperative über Verdinglichungseffekte in die Lebenswelt eindringen. Deren Ausgangspunkt ist also die Kolonialisierung der Lebenswelt durch die funktional ausdifferenzierten Systeme. Habermas hatte zwischen Emanzipations- und Rückzugspotentialen in „,neuen sozialen Bewegungen“" unterschieden; und freilich kommt für ihn v.a. ersteren besonderer Stellenwert zu, insofern sie es sind, die die verschütteten Protest- und Kritikpotentiale eines Klassenbewusstseins in neuer Form wieder aufleben lassen, also i.g.W. auf einen Antagonismus gegenüber „herrschenden Verhältnissen“ festgelegt sind.

Auch für Niklas Luhmann sind Ausgangspunkt sozialer Bewegungen Folgeprobleme funktionaler Differenzierung. Protestbewegungen hätten mit Individuen zu tun, ,[...] die die Zumutungen ihrer Lebenslage als paradox empfinden [...]" (Luhmann 1996, S. 203); die Gestik der Gesellschaftskritik und die Form des Protests diene im Wesentlichen dazu ,[...] Gleichgesinnte zu erkennen und entsprechende Sympathien zu bilden.“ (ebd., S. 207) Aber freilich sind für ihn weder die Ursachen, noch die Folgen der Formierung sozialer Bewegungen mit soziologischer Gesellschaftskritik direkt zu verknüpfen. Soziologinnen können die Kritik in der Gesellschaft beobachten, beschreiben und gegebenenfalls auch erklären, aber sich diese keinesfalls zu eigen machen. Ein Anknüpfungspunkt für Protest in der Gesellschaft ist die mit funktionaler Differenzierung entstandene Komplexität. Aber da man gegen Komplexität schwerlich protestieren könne, richtet der Protest sich vornehmlich an Funktionssysteme, die Zentren ausbilden, in unseren Fällen an die Politik.

Aber damit sind nur zwei Pole markiert, innerhalb deren die gegenwärtige Diskussion um Gesellschaftskritik und Soziologie bzw. Gesellschaftskritik und Gesellschaftstheorie sich bewegt. Eine, freilich etwas polemische Unterscheidung vorfindlicher „klassischer Kritiktypen“ hat Armin Nassehi jüngst vorgenommen und ihnen jeweils Fiktionen einer Beschreibbarkeit der Welt innerhalb der je eigenen Logik zugeordnet (vgl. Nassehi 2015, S. 115 f.) um sich in der Folge von allen mit einem eigenen Vorschlag zu einem „,neuen Kritiktypus“ davon abzugrenzen: 
- Linke Umbauperspektiven durch die Fixierung auf den einen Hauptwiderspruch in der Kapitalismuskritik, getragen von der Annahme einer objektivierbaren Analyse von Klassen- und Interessenlagen, deren reale Pendants dann als falsches Bewusstsein erscheinen;

- die bürgerlich-konservative Idee der moralischen Einsicht, des Verzichts auf Handlungsoptionen und der kollektivierbaren Moralität, die sich dann darüber wundert, dass sich Akteure entweder nach anderen Algorithmen verhalten oder aber das individuelle Motiv nicht kollektivierbar ist;

- schließlich die rechte Idee der Überwindung moderner Polykontexturalität durch die Simulation einer kulturellen/ethnischen/nationalen Einheit, die durch Einschluss- und Ausschlussroutinen die Komplexität der Situation zu überwinden trachtet - und am Ende feststellen muss, wie polykontextural selbst eine ethnisch völlig homogene Gesellschaft wäre.

Einen direkten Zusammenhang zwischen Gesellschaftskritik und Soziologie gibt es nur im ersten Fall. Bezogen auf die Gründungsphase der Soziologie hat Andreas Balog betont, dass die Verbindung von Gesellschaftskritik und Gesellschaftstheorie ,eher ein historisch erklärbarer Zufall“, jedoch keine sachlich bedingte Notwendigkeit sei. Denn „die Maßstäbe der Gesellschaftskritik" seien der Theorie gegenüber vielmehr autonom. (vgl. Balog 1993, S. 9 und 19)9 Daran anknüpfend argumentiert Evelyn Gröbl-Steinbach Schuster, es gebe grundsätzlich zwei Möglichkeiten einer Verbindung mit der Soziologie:

„Entweder sie erfolgt im Rahmen von Gesellschaftstheorie als Theorie eines zusammenhängenden Ganzen oder in Verbindung mit Soziologie, die sich als empirisch verfahrende Wissenschaft von sozialen Phänomenen sowie ihren Zusammenhängen und Veränderungen begreift. Über diese können mehr oder weniger weit reichende Hypothesen formuliert werden.“ (Gröbl-Steinbach Schuster 2012, S. 229)

Den ersten Weg hat die Kritische Theorie eingeschlagen; der zweite ist stärker in der Tradition der französischen Soziologie verankert, kann sich aber - mit anderer Akzentuierung - auch mit der Systemperspektive verbinden. Diese steht in engem Zusammenhang mit den durch funktionale Differenzierung entstandenen polytexturalen Perspektiven. Ein neuer Kritiktypus müsste mit der Widerständigkeit der Gesellschaft rechnen, also damit, dass sich Kritik nicht linear auf

9 Ihm zufolge weist Soziologie von vorne herein aber eine normative Dimension auf, und zwar als Folge der handlungsbegrifflichen Definition sozialer Phänomene. (vgl. Ebd., S. 24 ff.) Er selbst hatte an Gesellschaftskritik als zentraler Dimension soziologischen Wissens festgehalten. 
das Kritisierte bezieht; d.h. mit einer Gesellschaft, ,[...] die nach ihrer vielfältigen Logik auf jegliche Form der Intervention reagiert.“ (Nassehi 2015, S. 279)

Die Diskussion um Gesellschaftskritik in der Soziologie hat in den letzten Jahren wieder einen Aufschwung erfahren, ebenso aber die Kritik daran. Ich werde im Folgenden drei Positionen besprechen, die sich dieses Themas wieder angenommen haben und sie anschließend mit der Diskussion um die behandelten sozialen Protestbewegungen verbinden. Vorerst konzentriere ich mich auf drei Perspektiven im Verständnis von Protest als Kritik, Gesellschaftskritik und der Rolle der Soziologie: Gesellschaftskritik in der jüngeren Kritischen Theorie (Jürgen Habermas, Axel Honneth u.a.m), in der am gesellschaftskritischen Potential der Theorie festgehalten wird (3.1); in der Soziologie der Kritik (Luc Boltanski, Georg Vobruba), in der eine klare Trennung zwischen Gesellschaftstheorie und Gesellschaftskritik vorgenommen wird (3.2); in der kritischen Soziologie der Kritik (Stephan Lessenich), in der der Versuch unternommen wird, die beiden Perspektiven doch wieder miteinander zu verknüpfen (3.3).

\subsection{Gesellschaftskritik in der Kritischen Theorie}

Es soll und kann hier nicht das Kritikverständnis der frühen Kritischen Theorie besprochen werden; die Frage ist vielmehr, wie es sich gegenwärtig darstellt. Die kommunikationstheoretische Wende bei Jürgen Habermas ist von Max Preglau (1987) eingehend rekonstruiert worden und er hat gezeigt, wie Habermas sich sowohl von der marxistischen Variante als auch von der an der Kritik der instrumentellen Vernunft orientierten Variante der älteren Kritischen Theorie abgewendet hat, aber gleichwohl die Verbindung von Gesellschaftstheorie und der Dimension moralisch-praktischer Rationalität aufrechterhalten wollte.

Eines der zentralen Anliegen von Habermas war, wie Preglau betont, ,[...] die Klärung der methodologischen und theoretischen Grundlagen einer kritischen Gesellschaftstheorie [die dem] Typus der Kritik entspricht.“ (Preglau 1987, S. 218) In „Erkenntnis und Interesse“ (Habermas 1973) war es darum gegangen, einen internen Zusammenhang zwischen verschiedenen Typen der Erkenntnis und der Praxis nachzuweisen und zu zeigen, dass das emanzipatorische Erkenntnisinteresse (gegenüber dem technischen und dem praktischen) ,[...] den Zusammenhang des theoretischen Wissens mit einer Lebenspraxis (sichert), d.h. einem ,Gegenstandsbereich', der unter Bedingungen systematisch verzerrter Kommunikation und einer scheinhaft legitimierten Repression erst entsteht.“ (Habermas 1973, S. 400 - Hervorhebung im Original) Insofern sei radikale Erkenntniskritik nur als Gesellschaftstheorie möglich. (vgl. ebd., S. 9) In der Theorie kommunikativen Handelns stelle der Zusammenhang von Theorie und Praxis sich differenzierter dar, weil die Wissenschaftstypen auf universelle Geltungsgründe zurückgeführt werden; auf Basis der neuen sprachtheoretischen Grundlagen der Zusammenhang von Theorie und Praxis in einen von Theorie, (handlungsentlastetem) Diskurs und (praktischem) Handeln 
überführt worden sei und somit zwischen Gegenstandskonstitution und Geltungsproblem deutlicher unterschieden werden könne. (vgl. Preglau 1987, S. 216-218)

Ansatzpunkte für die (damals) neuen Protestpotentiale sind Prozesse der Verdinglichung, die sich als Pathologien der Lebenswelt niederschlagen. „Privatsphäre und Öffentlichkeit“ zeigten sich , ,...] im Lichte einer rationalisierten Lebenswelt, in der Systemimperative mit eigensinnigen kommunikativen Strukturen zusammenprallen." (Habermas 1981, S. 575) Widersprüche und Gegentendenzen entstünden nunmehr ,[...] in den Bereichen der kulturellen Reproduktion, der sozialen Integration und der Sozialisation $[\ldots]$ “ und werden ,[...] in subinstitutionellen, jedenfalls außerparlamentarischen Formen des Protestes ausgetragen.“ (ebd., S. 576) Es geht den Protestbewegungen demnach um den Widerstand gegen die Kolonialisierung der Lebenswelt und folgerichtig entstünden ,[...] die neuen Konflikte [...] an den Nahtstellen zwischen System und Lebenswelt.“ (ebd., S. 581; vgl. Preglau, S. 242) Als emanzipatorische soziale Bewegungen und Protestpotentiale hatte Habermas vor allem die Frauenbewegung genannt und den anderen einen stärker defensiven Charakter zugeschrieben, einschließlich der Grünen, der Alternativund Friedensbewegung, der Psychoszene, aber etwa auch dem Fundamentalismus u.a.m. Widerstands- und Rückzugspotentiale seien auf die Eindämmung formal-organisatorischer zugunsten kommunikativ-strukturierter Handlungsbereiche gerichtet. (vgl. Habermas 1981, S. 578 ff.)

In der Theorie des kommunikativen Handelns hat Habermas die normative Begründung einer kritischen Gesellschaftstheorie zwar fallen gelassen, sie aber auf die Ebene der kritisierbaren Geltungsansprüche transferiert:

„Die Gesellschaftstheorie braucht sich der normativen Gehalte der bürgerlichen Kultur, der Kunst und des philosophischen Denkens nicht mehr auf indirektem Wege, nämlich ideologiekritisch zu versichern; mit dem Begriff der kommunikativen, in den verständigungsorientierten Sprachgebrauch eingelassenen Vernunft [hätten sich drei Vernunftmomente herauskristallisiert - in] Wahrheitsfragen, in Fragen der Gerechtigkeit oder des Geschmacks." (Habermas 1981, S. 583 und f.)

Die Wissenschaften der Moderne bedürften keiner transzendentalen Begründung mehr, es könne auf die formale Pragmatik verwiesen werden.

„Die Theorie des kommunikativen Handelns zielt ja auf jenes Moment von Unbedingtheit, welches mit den kritisierbaren Geltungsansprüchen in die Bedingungen der Konsensbildungsprozesse eingebaut ist - als Ansprüche transzendieren diese alle räumlichen und zeitlichen, alle provinziellen Beschränkungen des jeweiligen Kontextes.“(ebd., S. 586 f.)

Um diesen Abschnitt argumentativ mit Max Preglau zu schließen: 
„Während Horkheimer u.a. in ihrer negativen Fixierung auf 'instrumentelle Vernunft' nicht im Stande waren, einen positiven Begriff nicht-verstümmelter Rationalität zu entwickeln und daher gezwungen waren, zwecks einer Explikation dieses Begriffs auf die [durch die positivistische ,Destruktion der Metaphysik' entwertete] philosophische Tradition der ,objektiven Vernunft' zurückzugreifen, hat sich Habermas mit seiner Theorie kommunikativen Handelns, [...] des vernünftigen Gehalts anthropologisch tiefsitzender Strukturen in einer rekonstruktiv ansetzenden Analyse vergewissert.“ (Preglau 1987, S. 243; inneres Zitat: Habermas 1981, S. $561 \mathrm{f})$

Es sei hier dahingestellt, ob diese tiefliegenden anthropologischen Strukturen sich auch empirisch nachweisen lassen (zur Kritik vgl. Dux 1986, 1987, 1998; Holz 1993; u.a.m.); für Habermas waren damit die normativ-praktischen Kriterien der Gesellschaftskritik in die Grundlagen der Gesellschaftstheorie eingebaut.

Auch für Axel Honneth „,...] soll die zentrale Zielsetzung der Kritischen Theorie, nämlich qua Theorie der Gesellschaft eine praktisch folgenreiche Kritik zu üben, weitergeführt werden.“ (Gröbl-Steinbach Schuster 2012, S. 239) Auch er will die Kritische Theorie umbauen, grenzt sich aber von der sprachpragmatischen Grundlegung ab und hält an der Notwendigkeit einer normativen Grundlage der Kritik fest, die bei den Anerkennungsansprüchen sozialer Akteurinnen ansetzt. (vgl. Honneth 1994) „Die Grundannahme der Anerkennungstheorie besagt nun, dass mit den empirisch nachweisbaren Anerkennungsansprüchen gleichzeitig ein normativer Bezugspunkt ausgezeichnet wird, der über das Faktische hinausweist [...] und [...] auf emanzipatorische Praxis ausgerichtet ist.“ (Gröbl-Steinbach Schuster 2012, S. 239) Gröbl-Steinbach Schuster konstatiert, dass es sich bei Honneth nicht um ein ,[...] Modell von politisch wirksamer Gesellschaftskritik [...]“ handelt, ,[...] sondern um Ideologiekritik als Kritik der Pathologien der gesellschaftlich herrschenden Vernunft.“ (ebd., S. 241) ${ }^{10}$ Die normativen Grundlagen, respektive moralische Ressourcen werden nicht (mehr) aus abstrakten Prinzipien, sondern aus der sozialen Praxis entwickelt. Es bleibt ihm aber letztlich, soziale Pathologien in den Sphären der Ökonomie sowie der Politik (und der Massenmedien) kritisch zu konstatieren. (vgl. dazu auch Honneth 2011)

\subsection{Soziologie der Kritik}

Im Rahmen eines Verständnisses der Soziologie als in erster Linie empirischer Wissenschaft, die aber an deren praktischen Dimension festhält, hat Luc Boltanski eine „Soziologie der Kritik" formuliert, in deren Zentrum die Praxis der sozialen Akteurinnen steht. Erst die pro-

10 „Kritik besteht konkret im Versuch, eine hermeneutische Wiederaneignung der unverzerrten Bedeutung moralischethischer Ideale zu bewirken, Praxis wird zur Vernunfttherapie der Subjekte durch die Theorie.“ (ebd.) 
fessionelle Distanzierungsleistung der soziologischen Akteurinnen aber ermögliche, vermittels der Beschreibung und Rekonstruktion der sozialen Realität, auch über diese hinauszudenken. Boltanski bestreitet eine interne Verbindung von Gesellschaftskritik und Soziologie, v.a. aufgrund deren nur bedingt prognosefähigen Charakters. (vgl. Gröbl-Steinbach Schuster 2012; Boltanski 2010) In einer pointierten Zusammenfassung:

„Luc Boltanski ist vermutlich der international prominenteste Protagonist einer Soziologie der Kritik, deren programmatische Grundüberzeugung darin besteht, dass Gesellschaftskritik nicht zur Stellenbeschreibung professioneller Soziolog/innen gehört, die als solche keineswegs und in keinerlei Hinsicht besonders qualifiziert oder gar legitimiert seien, um normative Aussagen darüber zu treffen, wie Gesellschaft idealerweise aussehen würde und was die Leute tunlichst tun, lassen oder wollen sollten. Aus der Perspektive einer Soziologie der Kritik sind es die sozialen Akteure selbst, die sich in der privilegierten und legitimierten Position befinden, um die soziale Welt, wie sie ist bzw. wie sie sich ihnen darstellt, zu kritisieren. Aufgabe der Soziologie ist es hingegen [...], die Menschen dabei zu beobachten, wie sie alltagspraktische Sozialkritik üben (wenn und soweit sie dies tun).“(Lessenich 2014, S. 16)

Darauf aber beschränkt Boltanski sich nicht wirklich. Es ist ihm sehr wohl ein Anliegen, einen Weg zwischen Kritischer Soziologie und (pragmatischer) Soziologie der Kritik aufzuzeigen (vgl. Boltanski 2010, S. 73 ff.). Dafür aber müssten an Metakritik (der Herrschaft) orientierte Soziolog/innen , , $[. .$.$] aus den Niederlagen der Vergangenheit Lehren ziehen und sich, [...] einen$ Analyserahmen vorgeben, mit dem sich die Beiträge jener beiden Programme [...] integrieren lassen.“ (ebd., S. 80) Sie müssten, verkürzt ausgedrückt, also einerseits die (totalisierende) Realität in Frage stellen und die Beherrschten mittels des eigenen Instrumentariums unterstützen, andererseits aber die kritischen Kompetenzen der handelnden Akteurinnen ernst nehmen. Die sozialen Akteurinnen seien letztlich durch Institutionen daran gehindert, in Distanz zur Realität zu gehen. „Diese Funktion der Institutionen klarzumachen - ebenso wie die Möglichkeit der Kritik darzulegen, d.h. zu zeigen, dass die Realität veränderbar ist - darin besteht die Aufgabe der Soziologie der Kritik.“ (Gröbl-Steinbach Schuster 2012, S. 237)

Diesen Perspektivenwechsel von einer kritischen Soziologie zu einer Soziologie der Kritik zuspitzend, hat Georg Vobruba die Position einer „politischen Soziologie der Gesellschaftskritik" formuliert. Ausgehend von der radikalen Erkenntniskritik im modernen wissenschaftlichen Weltbild und den Erkenntnisbedingungen der Soziologie, untrennbar mit dem Verlust absolutistischer Begründungen verbunden, nimmt Vobruba nunmehr die Position eines Beobachters zweiter Ordnung ein: „Wenn sich kein gesellschaftsexterner Punkt für Gesellschaftsbeobachtung, -analyse und -kritik mehr denken lässt, dann ist alle Kritik an der Gesellschaft Kritik in der Gesellschaft.“ (Vobruba 2009, S. 70) Beim Übergang von Kritischer Theorie zu einer Soziologie der Kritik handle es sich ,[...] um die zwingende Konsequenz des Strukturwandels des 
Denkens von der absolutistischen Logik zur Moderne.“ (Vobruba 2013, S. 155) ${ }^{11}$ Auch Gesellschaftskritik lasse sich mithin als soziales Phänomen verstehen, ,[...] das aus spezifischen Formen der Beobachtung und der Interpretation von Gesellschaft besteht, an die potentiell Handeln relevanter Akteure anschließt.“ (ebd., S. 160 und vgl. f.) Es geht ihm also darum, soziologisch zu beobachten, wie Gesellschaft in der Gesellschaft kritisch beobachtet wird und so könnten an die Gesellschaftskritik die klassischen Fragen der Soziologie gerichtet werden - in Bezug auf die Konstitutionsbedingungen von Kritik an der Gesellschaft, den Entstehungszusammenhang von Kritik und den Bedingungen für das Praktizieren von Kritik und in Bezug auf die ,[...] sich institutionell eröffneten Situationen, die das Auftreten von Kritik und ihren Konsequenzen praktisch möglich und somit empirisch wahrscheinlich machen." (Vobruba 2013, S. 162) Das kritische Potential einer, dergestalt empirisch ansetzenden, Soziologie der Kritik sieht Vobruba darin ,,...] nach Manifestationen von Gesellschaftskritik als nicht-intendierte Effekte institutioneller Entwicklungen zu fragen.“ (ebd., S. 163) Letztlich geht es ihm also auch hier um das Ausloten von Autonomiegewinnen der handelnden Akteure in der Gesellschaft, die ihrerseits durch institutionelle Arrangements eröffnet worden sind (vgl. dazu bereits Vobruba 1997 und 2009) und somit um die Bedingungen der Möglichkeit praktischer Gesellschaftskritik.

\subsection{Kritische Soziologie der Kritik}

Stephan Lessenich hat, wie schon Boltanski, auf beide Varianten der Kritik in der Soziologie - als Kritische Soziologie und als Soziologie der Kritik - und deren paradigmatisch-methodologischen Dissens reagiert und ihnen eine dritte Position gegenübergestellt. Diese ist m.E. jener Boltanskis näher, wenngleich der Text, auf den ich mich beziehe, nicht zuletzt als Antwort auf Georg Vobruba formuliert worden ist. Lessenich hatte sich aber auch schon zuvor, im Rahmen der Debatte mit Klaus Dörre und Hartmut Rosa, ähnlich positioniert und Gesellschaftskritik im Sinne einer Synthese von Künstler- und Sozialkritik als „riskante Soziologie“ bestimmt. (vgl. Lessenich 2009) Angesichts der Notwendigkeit einer soziologischen Ergründung der zentralen sozialen Frage der europäischen Gegenwartsgesellschaften bedürfe es einer „kritischen Soziologie der Kritik“, zumal eine in der Gesellschaft durchaus verbreitete und wohlbegründete alltagspraktische Gesellschaftskritik sich als hilf- und wirkungslos erweist und ,[...] gegen die ins Kraut schießende Kritik an den gesellschaftlichen Verhältnissen offensichtlich doch immer wieder ein politisches Kraut gewachsen ist.“ (Lessenich 2014, S. 9) Lessenich macht einen Aufriss der „Lage der arbeitenden Klassen in Europa zu Beginn des 21. Jahrhunderts“, d.h. er zeichnet kurz Einkommensverteilungen, Raten der Jugendarbeitslosigkeit, den Zusammenhang von Armut und Lebenserwartung in der Europäischen Union nach, um zu belegen, dass die

11 An mehreren Stellen übt Vobruba auch Kritik an Boltanskis Soziologie der Kritik, aber diese läuft m.E. ins Leere, als sie auf seinen Interpretationen beruht, nicht auf Boltanskis Intentionen (vgl. ebd.; Boltanski 2010). 
Konjunktur soziologischer Gesellschaftskritik schon ihre guten Gründe habe. Gegenüber Boltanski und Vobruba betont er, es brauche ,[...] wohl doch noch ein Drittes, um mit einer Soziologie der Gesellschaftskritik aufs Ganze zu zielen und zu gehen [...]“ (ebd., S. 19). Die Frage nach der Folgenlosigkeit der alltagspraktischen Kritik müsse in die soziologischen Fragen nach den Bedingungen der Möglichkeit von Gesellschaftskritik einbezogen werden. Die Aufgabe der Kritik könne man nicht allein den Leuten überlassen. Dabei gehe es weder um eine Kritik falschen Bewusstseins, noch um Ideologiekritik.

„Kritische Soziologie klärt die ,Gesellschaft der Leute“ (Vobruba 2009) darüber auf, dass diese selber gleichsam als Kleinsttriebwerke daran beteiligt sind, eben jenen gesellschaftlichen Wandel zu verhindern, den sie im Lichte einer Soziologie der Kritik besehen durchaus befürworten.“ (ebd., S. 21)

Eine kritische Soziologie der Gegenwartsgesellschaft habe vielmehr, durchaus im von Boltanski so bezeichneten, programmatischen Anliegen der Soziologie der Gesellschaftskritik zu folgen, ohne die alten Fehler zu begehen, sich in eine allzu große emotionale Nähe oder eben in eine intellektuelle Distanz zu den handelnden Akteuren zu begeben. „Es gilt mithin, analytisch das Bewusstsein für die systemischen Verstrickungen individueller Existenz und zugleich für die individuellen Handlungsspielräume in institutionellen Kontexten zu schärfen.“ (Lessenich 2014, S. 22; vgl. auch Lessenich 2014 a.)

\section{Schluss}

Was ist mit diesen unterschiedlichen Kritikperspektiven im Hinblick auf die soziologische Analyse der neuesten Protestbewegungen gewonnen? An deren Beschreibungen besteht ja kaum ein Mangel und es lassen sich - sowohl für den „Arabischen Frühling“ wie für Pegida, Legida etc. bei aller Kontingenz der Anlässe eine Vielzahl von Gründen benennen, die deren Entstehen sowie deren Dynamik begünstigt haben. Es lassen sich auch eine Reihe von institutionellen, politischen, demographischen, sozialen und kulturellen Bedingungen angeben, die uns letztlich auch zu deren Ursachen vordringen lassen. Kritische Soziologie, die sich i.g.W. immer schon an der Seite der Gesellschaftskritik der ,guten Gründe“ und den Widerstandspotentialen gegenüber verdinglichten, undemokratischen, technokratischen, ökonomistischen Strukturen befindet, kann die Jugendbewegung im arabischen Raum nur als emanzipatorische Bewegung begreifen und die rechtspopulistische Protestbewegung aus denselben guten Gründen, aber einem letztlich doch normativen Standpunkt kritisieren. Eine Soziologie der Kritik, wenn sie denn wirklich empirisch ist, kann in beiden Fällen die institutionellen Grundlagen sowie die sich eröffnet habenden Autonomiespielräume als Basis für deren Gesellschaftskritik aufklären, müsste sich 
aber freilich beiden gegenüber gleichermaßen neutral verhalten. Eine kritische Soziologie der Kritik würde in aller Eloquenz darauf verweisen, dass es sich im Falle der rechtspopulistischen Kritik zwar nicht unbedingt um einen Verblendungszusammenhang handelt, das Soziologinnenherz aber doch eher links schlägt.

Wenn sich aber für Gesellschaftskritik in der Soziologie und in der Gesellschaftstheorie erst recht kein Anhalt mehr finden lässt und wenn - anders als in der kritischen Perspektive der Theorie des kommunikativen Handelns, die letztlich auf empirisch nicht belegbaren anthropologischen Tiefenstrukturen angelegten - auch normativ-praktische Kriterien sich nicht mehr mit der Soziologie als professionalisierter Wissenschaft verbinden lassen, dann geraten wir als Soziologinnen angesichts der beschriebenen, widersprüchlichen Protestbewegungen zumindest ins Dilemma forschungsstrategischer Entscheidungen: Welchem Typus von Gesellschaftskritik wenden wir uns aus der Perspektive einer Soziologie der Kritik zu, müssen wir uns nicht doch von spezifischen Erkenntnisinteressen leiten lassen? Reicht es hin, in vergleichenden Untersuchungen Typologien von Protestbewegungen zu erstellen und inwieweit setzen wir uns damit erst recht dem Vorwurf der Parteilichkeit oder eben jenem der mangelnden gesellschaftlichen Verantwortung aus? Welche Kriterien stehen uns zur Verfügung bzw. reichen hin, um derartige Typologien trennscharf erstellen zu können? Wenn wir also weder in der Wert- oder Moralsphäre angesiedelte und schon erst recht nicht in der Wissenschaft verankerte Begründungen und somit Bezugspunkte für Kritik in Anschlag bringen können, dann bleibt uns nichts anderes übrig, als sie in der Faktizität der Verhältnisse zu suchen. Hier kommt aber als einzige nichtideologische verzerrte die Errungenschaft der in der Rechts- und Verfassungsordnung festgeschriebene Universalität der Menschenrechte und deren Unverletzlichkeit in Frage. Aber setzen wir uns nicht - um es polemisch zu sagen - damit der Kritik aus, die auch an Hegels Mythologem des im absolutistischen Staat verwirklichten Weltgeistes gerichtet gewesen war? Der kritische Stachel im historisch gewordenen Fleisch der Soziologie und der Soziologinnen hinterlässt zumindest Unbehagen angesichts einer praktischen Gesellschaftskritik, die zumindest partiell als Negation historisch-normativer Errungenschaften auftritt und die wir uns so wirklich nicht vorgestellt haben.

\section{Literatur}

Assheuer, Thomas. 2014. Die nationale Querfront. In: ZEIT ONLINE 2014/52. http://www.zeit.de/2014/52/ pegida-proteste-links-rechts (letzter Zugriff am 29.04.2016)

Balog, Andreas. 1993. Soziologie, Gesellschaftstheorie und Gesellschaftskritik. In: Soziologie und Gesellschaftskritik. Beiträge zum Verhältnis von Normativität und sozialwissenschaftlicher Analyse, hrsg. Andreas Balog und Johann August Schülein, Österreichische Zeitschrift für Soziologie, Sonderband 2. Wien: VWGÖ-Verlag: 9-36. 
Bergmann, Werner. 1987. Was bewegt die soziale Bewegung? Überlegungen zur Selbstkonstitution der „neuen“ sozialen Bewegungen. In: Theorie als Passion. Niklas Luhmann zum 60. Geburtstag, hrsg. Dirk Baecker, Jürgen Markowitz und Rudolf Stichweh, 362-393. Frankfurt am Main: Suhrkamp.

Boltanski, Luc. 2010. Soziologie und Sozialkritik. Frankfurter Adorno-Vorlesungen 2008. Frankfurt am Main: Suhrkamp.

Courbage, Youssef und Emmanuel Todd. 2008. Die unaufhaltsame Revolution. Wie die Werte der Moderne die islamische Welt verändern. München/Zürich: Piper.

Dörre, Klaus, Stephan Lessenich und Hartmut Rosa. 2009. Soziologie. Kapitalismus. Kritik. Eine Debatte. Frankfurt am Main: Suhrkamp.

Dux, Günter. 1986. Kommunikative Vernunft und Interesse. Zur Rekonstruktion der normativen Ordnung in egalitär und herrschaftlich organisierten Gesellschaften. In: Kommunikatives Handeln. Beiträge zu Jürgen Habermas' , ,Theorie des kommunikativen Handelns“, hrsg. Axel Honneth und Hans Joas, 110-143. Frankfurt am Main: Suhrkamp.

Dux, Günter. 1987. Natur - Gesellschaft - Geist. Zur soziologischen Anthropologie der Erkenntnis. In: Archiv für Kulturgeschichte 69: 196-229.

Dux, Günter. 1998. Anthropologie als Grundlagenwissenschaft. In: Freiburger Universitätsblätter, Heft 139: 9-19.

Geiges, Lars, Stine Marg und Franz Walter. 2015. Pegida. Die schmutzige Seite der Zivilgesellschaft? Berlin: Transscript.

Gerlach, Julia. 2016. Fünf Jahre Arabellion: Das Ende eines Traums? In: Blätter für deutsche und internationale Politik 2/2016: 47-56.

Gröbl-Steinbach Schuster. 2012. Der Standpunkt der Gesellschaftskritik. In: Handlung. Neue Versuche zu einem klassischen Thema, hrsg. Johann August Schülein und Gerhard Mozetic, 223-245. Wiesbaden: Springer VS.

Habermas, Jürgen. 1973. Erkenntnis und Interesse. Frankfurt am Main: Suhrkamp.

Habermas, Jürgen. 1981. Theorie des kommunikativen Handelns. 2 Bände. Frankfurt am Main: Suhrkamp.

Holz, Klaus. 1993. Historisierung der Gesellschaftstheorie. Zur Erkenntniskritik marxistischer und kritischer Theorie. Pfaffenweiler: Centaurus.

Honneth, Axel. 1994. Kampf um Anerkennung. Frankfurt am Main: Suhrkamp.

Honneth, Axel. 2011. Das Recht der Freiheit. Grundriß einer demokratischen Sittlichkeit. Berlin: Suhrkamp.

Jaeggi, Rahel und Tilo Welsch (hrsg.) 2009. Was ist Kritik? Frankfurt am Main: Suhrkamp

Lessenich, Stephan. 2009. Künstler- oder Sozialkritik? Zur Problematisierung einer falschen Alternative. In: Soziologie, Kapitalismus, Kritik. Eine Debatte, hrsg. Klaus Dörre, Stephan Lessenich und Hartmut Rosa, 224-242. Frankfurt am Main: Suhrkamp.

Lessenich, Stephan. 2014. Soziologie - Krise - Kritik. Zu einer kritischen Soziologie der Kritik. In: Soziologie, 43. Jg., Heft 1: 7-24. 
Lessenich, Stephan. 2014a. Akteurszwang und Systemwissen: Das Elend der Wachstumsgesellschaft. In: Systemzwang und Akteurswissen. Theorie und Empirie von Autonomiegewinnen, hrsg. Thilo Fehmel, Stephan Lessenich und Jenny Preunkert. Frankfurt am Main: Campus Verlag.

Luhmann, Niklas. 1996. Protestbewegungen. In: Protest. Systemtheorie und soziale Bewegungen, hrsg. Kai-Uwe Hellmann, 201-215. Frankfurt am Main: Suhrkamp.

Nassehi, Armin. 2015. Die letzte Stunde der Wahrheit. Warum rechts und links keine Alternativen mehr sind und Gesellschaft ganz anders beschrieben werden muss. Hamburg: Murmann Publishers.

Nordhausen, Frank. 2011. Ägypten: Die Sieger des Tahir-Platzes. In: Die arabische Revolution, hrsg. Frank Nordhausen und Thomas Schmid, 37-62. Berlin: Christoph Links Verlag.

Perthes, Volker. 2011. Der Aufstand. Die arabische Revolution und ihre Folgen. München: Pantheon Verlag.

Preglau, Max. 1987. Wachstumskrise und Gesellschaftstheorie. Krisenanalyse am Besipiel der Frankfurter Schule. Frankfurt/New York: Campus.

Roy, Olivier. 2011. Post-Islamic Revolution. In: European Institute February 2011:1-4. http://www. europeaninstitute.org/index.php/ei-blog/119-february-2011/1238-qpost-islamic-revolutionqevents-in-egypt-analyzed-by-french-expert-on-political-islam (letzter Zugriff am 29.04.2016).

Rucht, Dieter. 2014. Kein Vertrauen mehr in die etablierte Politik. Dieter Rucht im Gespräch mit Christoph Schmitz. In: Deutschlandfunk: http://www.deutschlandfunk.de/soziologe-ueber-pegida-kein-vertrauen-mehr-in-die.691.de.html?dram:article_id=306345 (letzter Zugriff am 29.04.2016).

Schmid, Thomas. 2011: Tunesien: Die Jasmin-Revolution. In: Die arabische Revolution, hrsg. Frank Nordhausen und Thomas Schmid, 15-36. Berlin: Christoph Links Verlag.

Ullrich, Peter. 2015. Postdemokratische Empörung. Ein Versuch über Demokratie, soziale Bewegungen und gegenwärtige Protestforschung. Berlin: Ipb working papers.

Vobruba, Georg. 1997. Autonomiegewinne. Sozialstaatsdynamik, Moralfreiheit, Transnationalisierung. Wien: Passagen Verlag.

Vobruba, Georg. 2009. Die Gesellschaft der Leute. Kritik und Gestaltung der sozialen Verhältnisse. Wiesbaden: VS Verlag für Sozialwissenschaften.

Vobruba, Georg. 2013. Soziologie und Kritik. Moderne Sozialwissenschaft und Kritik der Gesellschaft. In: Soziologie, 42. Jg., Heft 2: 147-168.

Vorländer, Hans. 2015. Wer geht warum zu PEGIDA-Demonstrationen? Präsentation der ersten empirischen Umfrage unter PEGIDA-Teilnehmern. In: Spiegel Online 14.01.2015: http://www.spiegel.de/ politik/deutschland/pegida-studie-in-dresden-marschiert-die-mittelschicht-a-1012913.html (letzter Zugriff am 29.04.2016).

Vorländer, Hans. 2015a. Was ist Pegida und warum? In: FAZ.NET. http:/www.faz.net/aktuell/politik/diegegenwart/protestbewegungen-was-ist-pegida-und-warum-13863310.html\#/elections (letzter Zugriff am 29.04.2016). 
Walter, Franz. 2015. Psychogramm der Pegida-Anhänger. In: Spiegel-Online vom 19.01.2015. http:// www.spiegel.de/politik/deutschland/pegida-franz-walter-legt-studie-zu-demonstranten-in-dresdenvor-a-1013688.html (letzer Zugriff am 29.04.2016).

WZB-Bericht (10 Autor/inn/en). 2015. Protestforschung am Limit. PDF-File in WZB: https://www.wzb. eu/sites/default/files/u6/pegida-report_berlin_2015.pdf (letzter Zugriff am 29.04.2016).

Gerda Bohmann, ist a. o. Professorin am Institut für Soziologie und Empirische Sozialforschung der Wirtschaftsuniversität Wien. Arbeitsgebiete/Forschungsschwerpunkte: Soziologische Theorie, historischgenetische Theorie, politische Religionen und Säkularisierung. Neuere Publikationen: Gemeinsam mit Heinz-Jürgen Niedenzu, Hrsg., 2014. Multiple Modernities - Chancen und Grenzen eines Konzepts. Themenheft. Österreichische Zeitschrift für Soziologie (ÖZS) (4). Gemeinsam mit Johanna Hofbauer und Johann August Schülein, Hrsg., Sozioökonomische Perspektiven. Texte zum Verhältnis von Gesellschaft und Ökonomie. Wien: facultas wuv. 2014. Wiederkehr der Religionen, postsäkulare Gesellschaft, oder doch weitergehende Säkularisierung? Widerspruch zu einem gängigen Diskurs. In: Systemzwang und Akteurswissen. Theorie und Empirie von Autonomiegewinnen, Hrsg. Thilo, Fehmel, Lessenich, Stephan, Preunkert, Jenny, 53-80. Frankfurt am Main: Campus. 2014. 